\title{
On the wave-front shape and the advancing of the wetting front of a dam-break flood over an inclined plane of arbitrary bottom slope
}

\author{
P. Bohórquez * \\ Universidad de Málaga, E.T.S. Ingenieros Industriales, Málaga, Spain
}

In this note we consider the analysis of the wave-front shape resulting from the sudden release of a finite volume of water over an inclined plane bed of arbitrary bottom slope. To that end we use the one-dimensional turbulent shallow water equations with a constant friction factor. We propose an asymptotic analytical solution for the height and velocity in the wave tip region based on the velocity of the wetting front and its temporal derivatives, in a similar fashion as Whitham (1955). For large time, an analytical solution is obtained for the advance of the wetting front. This proposal is compared and validated with a numerical simulation computed with a second-order TVD-MinMod numerical scheme. The divergence of the Hunt's solution (Hunt 1982; Hunt 1984) for the advancing of the wetting front as time increases is also established. Our solution for the tip region is tested against the numerical one, and is also compared with that deduced by Hunt (1984) for small slopes of the bottom - a really good agreement is found.

\section{INTRODUCTION}

As far as the author knows the first theoretical studies on the effect of hydraulic resistance on dam-break waves were performed by Dressler (1952) and Whitham (1955) - these investigations started with the analysis of horizontal beds. Later on more realistic problems were analysed, for instance dam-break floods of a finite volume of water over plane inclined beds [i.e., Hunt $(1982,1984)]$. Recently this topic have achieved new interest and insight in its theoretical aspect [see, for instance, Hogg \& Pritchard (2004)] as well as in its experimental version (Lauber 1997).

However, the above theoretical works used the general expressions of Saint Venant, often called the nonlinear shallow-water equations, valid only for small slopes of the bottom. In this line we have considered the analysis of the release of a finite volume of water over an inclined bed of arbitrary bottom slope, using the expressions provided by Fernandez-Feria (2006) (but adding basal drag effects) to appropriately describe the fluid movement over arbitrary but constant slopes.

The paper is organized as follows: we firstly formulate the problem $(\S 2)$. We then analyse the flow movement under the kinematic wave approximation and obtain the velocity and height field as well as the wave-front location ( $\S 3.1)$. In $\S 3.2$ we investigate

${ }^{*}$ This research has been supported by a fellowship from the Ministerio de Educación y Ciencia of Spain (AP-2004-3719) 

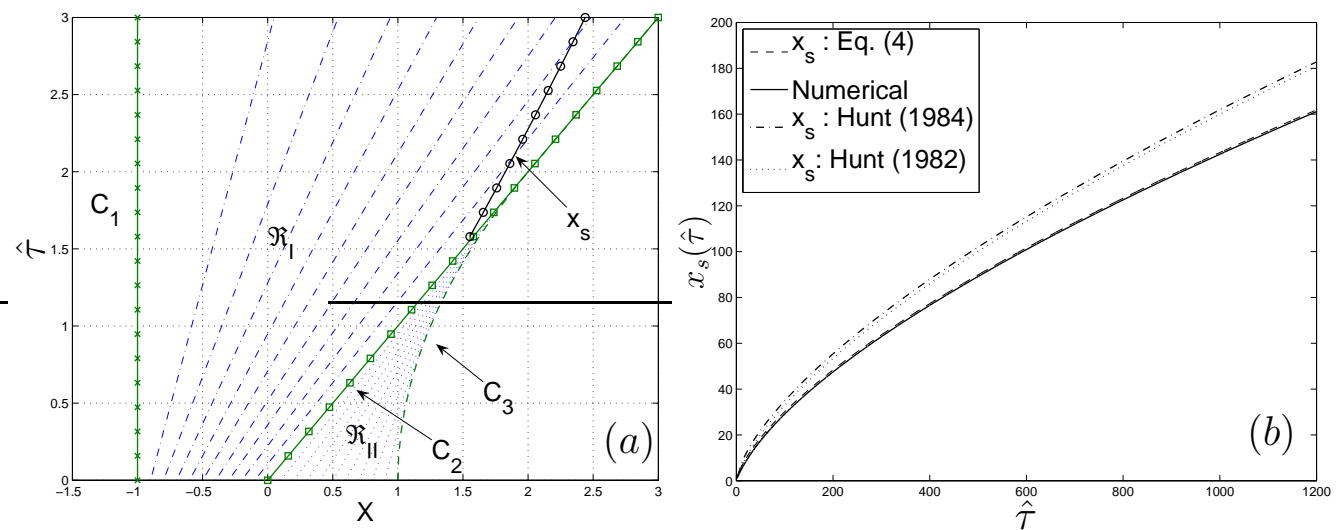

Figure 1: (a) Characteristic curves in a space-time diagram under the kinematic wave approximation for $e=1$. (b) Temporal evolution of the wave-front obtained numerically compared with the asymptotic result by Hunt $(1982,1984)$ and with Equation (4); we have selected $\eta_{0}=1 m, e=1$ and $F r_{e q} \equiv \sqrt{8 e / f}=2$.

the wave shape in the tip region and provide the solution as an asymptotic expansion, indeed the explicit expressions for the whole coefficients are deduced. This solution is also shown to be in close agreement with Hunt's solution (Hunt 1984) for small slopes of the bottom. We compare our theoretical predictions with numerical experiments in $\S 3.1$ and $\S 3.2$. A summary and some concluding comments are given in $\S 4$.

\section{FORMULATION OF THE PROBLEM}

We consider here the one-dimensional flow over a constant slope bed. In the shallow-water approximation the dimensionless equations for the mass conservation and momentum in the direction of the flow can be written as

$$
\partial_{t} \eta+\partial_{X}(\eta U)=0, \quad \partial_{t} U+U \partial_{X} U+\cos \theta \partial_{X} \eta=\sin \theta-f U^{2} /(8 \eta) .
$$

The notation and initial conditions of the problem under consideration are exactly the same as used in Fernandez-Feria (2006), therefore we do not repeat this information. We have only added the effects of basal drag using the Darcy-Weisbach friction factor $f$ (considered constant in the present work) in the momentum balance equation. These equations, are valid for any slope $\theta$ of the constant-slope bed, not just for small channel slope, provided that the ratio of the depth $\eta$ to the streamwise extent is sufficiently low.

\section{RESULTS}

\subsection{Kinematic wave solution}

In this section we follow the same theoretical approach as Hunt (1982) but solving Equations in (1) valid for arbitrary bottom slope to obtain the forward wave (wetting front) advancing over a dry bed.

In the kinematic wave approximation, one neglects the left hand side of the momentum equation and obtains the relation that follows between $\eta$ and $U, U^{2}=(8 \eta / f) \sin \theta$. Taking into account this relationship, mass conservation yields

$$
\frac{\partial \eta}{\partial \tau}+\frac{3}{2} \sqrt{\eta} \frac{\partial \eta}{\partial X}=0, \quad \tau \equiv\left(\frac{8 \sin \theta}{f}\right)^{1 / 2} t
$$

The equation above can be readily integrated together with the initial condition for $\eta$ [see Fernandez-Feria-(2006) for its definition]. The wave profile should be defined in two different regions: the first area (denoted by $\Re_{I}$ ) is enclosed by the two real characteristics 


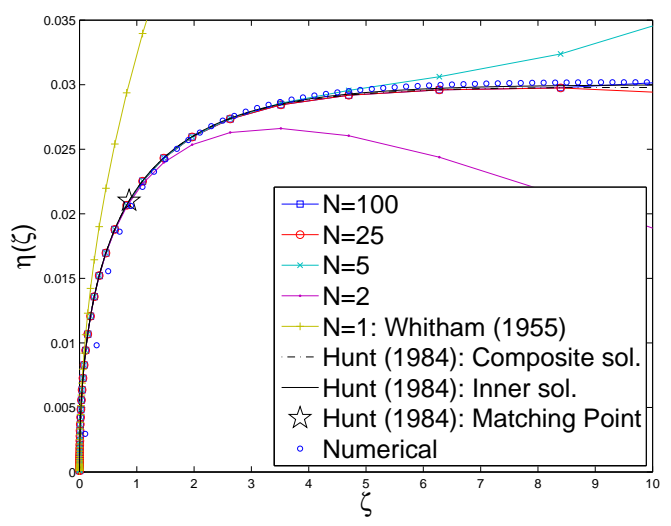

Figure 2: Asymptotic solution (7) for the height $\eta(\zeta)$ for a given instant of time for several values of $N$. The case $N=1$ corresponds to the analogous solution given by Whitham (1955). Also included is the asymptotic solution by Hunt (1984) and the numerical one for the same conditions. We have selected $t=4411, \eta_{0}=1 \mathrm{~m}, e=0.0175$ and $F r=2.5$.

$C_{1} \equiv-1 / e$ and $C_{2}(\tau) \equiv 3 \tau / 2$, and this region exists for any $\tau$; the second region $\left(\Re_{I I}\right)$ ranges between the characteristic $C_{2}(\tau)$ and $C_{3}(\tau) \equiv\left(16 e^{2}+9 \tau^{2}\right) /(8 e)$, and it exists just for $\tau \leq 4 e / 3$. After defining these two different areas the solution reads

$$
\eta(X, \tau)=\left\{\begin{array}{ll}
1+(e / 2)\left(e \hat{\tau}^{2}+2 X-\hat{\tau} \sqrt{4+e^{2} \hat{\tau}^{2}+4 e X}\right) & \text { if } X \in \Re_{I} \\
1-\left(2 e X-\hat{\tau}^{2}-\hat{\tau} \sqrt{4 e^{2}+\hat{\tau}^{2}+4 e X}\right) /\left(2 e^{2}\right) & \text { if } X \in \Re_{I I}
\end{array},\right.
$$

being $\hat{\tau} \equiv 3 \tau / 2$. As in Hunt's (1982) solution, a shock must be inserted in the solution to satisfy the mass conservation requirement. For simplicity we provide the location of the shock $x_{s}(\tau)$ just for $\tau>3 x_{s} / 2$. In closed-form it is given by

$$
\hat{\tau}\left(4+e^{2} \hat{\tau}^{2}+4 e x_{s}\right)^{3 / 2}=e^{3} \hat{\tau}^{4}+12 x_{s}+6 e^{2} \hat{\tau}^{2} x_{s}+6 e\left(\hat{\tau}^{2}+x_{s}^{2}-1\right) .
$$

Figure 1a depicts both the region $\Re_{I}$ (dashed-dotted line) and the region $\Re_{I I}$ (dotted line) in the characteristic plane $\{X, \hat{\tau}\}$ for $e=1$. The border between these two regions is established by the characteristic curve $C_{2}$ (solid, squared line). One should note the presence of the shock $x_{s}$ (solid, circled line), which establishes the validity of the solution (3): on the right hand side of the shock the solution is no longer valid, and both the height and the velocity field vanish. The other two characteristics $C_{1}$ (solid, crossed line) and $C_{3}$ (dashed line) are also shown in the same figure. To validate the front location (4), Equations in (1) (with the corresponding initial conditions) have been solved numerically on an uniform grid using an upwind TVD (total variation diminishing) method, secondorder accurate in both space and time, with semi-implicit and upwind treatment of the source terms, as described by Burguete \& Garcia-Navarro (2001). The boundary condition used for the wetting front is a cut-off height $\left(\eta / \eta_{\max }<10^{-4}\right)$, the Courant-Friedrich-Lewy number is $C F L=0.45$, and the number of nodes is $n x=10^{4}$. Figure $1 \mathrm{~b}$ shows a close agreement between the numerical (solid line) and the analytical result (4) (dotted line). We have also plotted the asymptotic result provided by Hunt $(1982,1984)$ considering the shallow-water equations due to Saint Venant. The solutions by this author becomes worse as time goes on because the slope $e$ is not small.

\subsection{Wave-front shape}

If we define the variable $\zeta=x_{f}(t)-X$, where $x_{f}$ is the position of the wetting front, Equation (1) read (note that $U>0$ )

$$
\partial_{t} \eta+\left(x_{f}^{\prime}-U\right) \partial_{\zeta} \eta-\eta \partial_{\zeta} U=0
$$




$$
\partial_{t} U+\left(x_{f}^{\prime}-U\right) \partial_{\zeta} U-\cos \theta \partial_{\zeta} \eta=\sin \theta-f U^{2} /(8 \eta),
$$

where primes denote differentiation with respect to $t$. One can obtain a solution of the resulting equations in powers of $\zeta$ using the same expansion as Whitham (1955), which can be written as

$$
\eta(\zeta, t)=\sum_{j=0}^{N} \eta_{j}(t) \zeta^{\frac{j+1}{2}}, \quad U(\zeta, t)=\sum_{j=-1}^{N} U_{j}(t) \zeta^{\frac{j+1}{2}} .
$$

If the wave-front is taken to be $X=x_{f}(t)$, then $U_{-1}=x_{f}^{\prime}$ at the wave-front. On the other hand, the particle acceleration, $\partial_{t} U+\left(x_{f}^{\prime}-U\right) \partial_{\zeta} U$, is expected to be finite while the effect of resistance is to pile up the fluid near the wave-front, so that $\partial_{\zeta} \eta$ becomes large. Therefore, to first approximation, $-\eta \cos \theta \partial_{\zeta} \eta=\eta \sin \theta-f U^{2} / 8$. Taking into account (7) and grouping terms of order $\zeta$ one obtains $\eta_{0}=x_{f}^{\prime} / 2(f / \cos \theta)^{1 / 2}$. Now, substituting the expansion (7) in the continuity equation (5) and grouping again terms of order $\zeta$ one obtains $U_{0}=0$. Repeating this process for the next exponents in $\zeta$, firstly with the momentum equation (6), and then with the mass balance (5), the coefficients $\eta_{i}$ and $U_{i}$ are obtained explicitly. For instance, grouping terms $O\left(\zeta^{1 / 2}\right)$ in the mass balance provides $\eta_{1}=2\left(x_{f}^{\prime \prime}-\sin \theta\right) /(3 \cos \theta)$. All the coefficients can be obtained analytically:

$$
\begin{gathered}
U_{N}=\frac{1}{\eta_{0}}\left(\frac{2}{N+2} \eta_{N-1}^{\prime}-\sum_{j=1}^{N-1} U_{j} \eta_{N-j}\right) \text { for } N \geq 1, \\
\eta_{N}=\frac{2}{N+2} \frac{1}{\eta_{0} \cos \theta}\left[-\cos \theta \sum_{j=1}^{N-1} \frac{N-j+1}{2} \eta_{j} \eta_{N-j}-\sin \theta \eta_{N-1}+\frac{f}{8} \sum_{j=-1}^{N-1} U_{j} U_{N-j-2}+\right. \\
\left.\sum_{j=0}^{N-1} \eta_{j} U_{N-2-j}^{\prime}+\sum_{j=0}^{N-1}\left(-\eta_{j} \sum_{k=1}^{N+1-j} \frac{N-k-j+1}{2} U_{N-k-j} U_{k-1}\right)\right] \text { for } N \geq 2 .
\end{gathered}
$$

This asymptotic solution, which actually converges even for $\zeta>1$ (see Fig. 2), was originally found by Whitham (1955), but this author only provided explicit expressions for the first few coefficients, just up to $N=1$. The first result we must point out is that the velocity field depends on $\zeta$ from the third term in the expansion, and therefore the assumption performed by Whitham "that $U$ is nearly uniform in the tip region" (Whitham $1955)$ is valid only until $O(\zeta)$. Second, the solution converges slowly as $N$ is increased if $\zeta$ is not too small, as it is shown in Figure 2, where the height $\eta$ has been plotted as a function of $\zeta$ for a given $t$. To compute $x_{f}^{\prime}$ and next derivatives we have used the previous result (4) valid for any slope of the bottom.

In that figure we also include the form of the wetting front given by the asymptotic solution by Hunt (1984) showing a very good agreement with the asymptotic solution (7)(9) if $N$ is sufficiently large. This is so because we have used a very small bottom slope (note that $e=0.0175$ implies $\theta=1^{\circ}$ ), and thus the Saint Venant equations for a plane bed approximates with precision (1), in order to check (7) with a known solution. But this solution is actually valid for any constant slope. Our expansion is in agreement with the inner solution (plotted in continuous line) for $N \geq 2$. From the matching point (big star) to the right hand side, the inner and the composed solution (dashed-dotted line) by Hunt are almost identical, and we need quite a lot terms in the expansion (e.g., $N>25$ ) to obtain a similar result. Furthermore, we have also computed the numerical solution with the same technique described in $\S 3.1\left(C F L=0.45\right.$ and $\left.n x=10^{4}\right)$ and a very good agreement has 
been found with the theoretical ones (Fig. 2). Finally, although the slope of the bed is small, the kinematic wave approximation is valid [one can verify $x_{s} \gg 4(e+1 / e)$ ], and thus the source terms in Equation (1) dominate the fluid movement. Even in this case the cut-off height method used to compute the advance of the wetting-front is good enough for the problem under consideration.

\section{CONCLUSIONS}

In this study we have considered the effects of hydraulic resistance on the dam-break flood over an inclined plane of arbitrary but constant slope.

For the kinematic wave regime the theoretical location of the wetting front has been supplied together with the velocity and height field $(\S 3.1)$. We have also solved the shallow-water equations (with the appropriate initial conditions) using an upwind TVD method, second-order accurate in both space and time, with semi-implicit and upwind treatment of the source terms. We find that both solutions are in agreement. One of the most noticeable effects of the results for large slopes of the bottom is the divergence of the previous solution by Hunt $(1982,1984)$, who provided a similar asymptotic solution using the traditional Saint Venant equations. This divergence arises mainly because the whole gravity $g$ moves the fluid when the Saint Venant equations are used, instead of its projection along the perpendicular to the plane.

We have also considered the analysis of the shape of the flood in the tip region (§3.2). The results are not only valid for a dam-break flood but also for any mass of fluid that spreads on a plane bed. The solution has been obtained as a full expansion in terms of the streamwise coordinate located at the wetting front. Our results are directly comparable to those obtained by Hunt (1984) for small slopes of the bottom. We find that the earlier result exhibits a good agreement with our solution when we use enough terms in the expansion, but Hunt's solution is valid only for small slopes of the bottom. The accuracy of the numerical solution has been also checked, and very good results are obtained by just using a cut-off height as boundary condition for the wetting front.

Finally, we note that the solutions developed could be used to check, for instance, the precision of other numerical schemes or boundary conditions.

\section{REFERENCES}

Burguete, J. \& Garcia-Navarro, P. (2001). Efficient construction of high-resolution TVD conservative schemes for equations with source terms: application to shallow water flows. Int. J. Num. Meth. Fluids 37(1), 209-248.

Dressler, R. (1952). Hydraulic resistance effect upon the dam-break functions. J. Res. Nat. Bur. Stand. 49, 217-225.

Fernandez-Feria, R. (2006). Dam-break flow for arbitrary slopes of the bottom. Journal of Engineering Mathematics 54(7), 319-331.

Hogg, A. J. \& D. Pritchard (2004). The effects of hydraulic resistance on dam-break and other shallow inertial flows. Journal of Fluid Mechanics 501, 179-212.

Hunt, B. (1982). Asymptotic solution for dam-break problem. J. Hydraul. Div. 108(1), $115-126$.

Hunt, B. (1984). Perturbation solution for dam-break floods. J. Hydraul. Eng. 110(8), $1058-1071$.

Lauber, G. (1997). Experimente zur Talsperrenbruchwelle im glatten geneigten Rechteckkanal. Ph. D. thesis, VAW-ETH, Zrich, Switzerland.

Whitham, G. (1955). The effects of hydraulic resistance in the dam-break problem. Proc. Roy. Soc. of London 227, 399-407. 